\title{
Influence of proprioceptive neuromuscular facilitation therapeutic exercise on woman with temporomandibular joint disorder: a case study
}

\author{
Byung-Ki Lee* \\ Department of Physical Therapy, Daewon University College, Jecheon, Korea
}

The purpose of this study was to determine the effect of therapeutic exercise for a woman with temporomandibular joint disorder. She was 31 year old and had limitation in the opening of mouth. When opening the mouth, she complained pain in the temporomandibular joint and noise. The health status of the patient was classified based on International Classification Functioning, Disability, and Health regarding the activity of the patient. The activity limitation was determined by the ability of opening the mouth, while the impairments in the body structure and body function were determined by the forward-headed posture due to the hypertension and shortening of upper cervical extensors. The proprioceptive neuromuscular facilitation (PNF) program consists of eight types of therapeutic exercises considering the alignment of the cervical spine and the pain in the temporomandibular joint. The application of the PNF program was composed of 12 sessions over 4 weeks and each session continued $30 \mathrm{~min}$. Before and after the application of the PNF program, the up-down length and the left-right width of the bite mark on the apple were measured (activity limitation test). The length of the apple that was bitten was measured using a tape measure (activity limitation test). The test on the abnormal alignment of the cervical spine was conducted using a plastic goniometer to measure the angle to the cervical spine (impairment test). After a 4-week PNF program, patient could open mouth wide without pain. The present results suggest that PNF program can be used to enhance the stability and mobility of the temporomandibular joint. With this, the impairment in the body structure and body function and activity of patient were improved.

Keywords: Temporomandibular disorders, Proprioceptive neuromuscular facilitation, International Classification Functioning, Disability, Health

\section{INTRODUCTION}

Temporomandibular disorder (TMD) causes pain around the temporomandibular joint and dysfunction of surrounding muscles. Also, the restraints in the movement of the mandible results in symptoms while chewing and noise at the joints. The causes of TMD can be attributed to many factors. These mainly include the pathological status of the muscles that are frequently used in chewing, the posture muscles of the neck, and the connection status of the Temporomandibular joints (El Hage et al., 2013).

The symptoms of TMD include excessive tension in the muscles in the chin, face, and neck, remitted movement of the chin, pain when opening or closing the mouth, and noise from the

Temporomandibular joint. Due to the damages in the body structure and body functional compromises, the patients experience difficulties in swallowing, eating, chewing in daily living life (Okeson, 2015). (Cortese et al., 2017) argued that the forward headed posture changes the position of the mandible, so that the mandible is pulled posteriorly and inferiorly, while the locations of the mandibular condyle and articular disc positions are changed in temporomandibular joint.

PNF was developed by (Kabat and Knott, 1953) during the 1950s. The therapy was designed for patients with paralyzed muscles and pain due to musculoskeletal diseases. In this method, the movement of the body is facilitated through contraction of the muscles through diagonal movement patterns of the limbs and

*Corresponding author: Byung-Ki Lee (iD https://orcid.org/0000-0002-1639-3318 Department of Physical Therapy, Daewon University College, 316 Daehak-ro, Jecheon 27135, Korea

Tel: +82-43-649-3224, Fax: +82-043-649-3690, E-mail: toppt@naver.com

Received: August 14, 2018 / Accepted: November 2, 2018 
the body trunk, so that the functions of the patients can be enhanced (Kabat and Kontt, 1953). PNF is a useful exercise in causing muscular contractions and movements of limbs without pain on the patients with musculoskeletal diseases. Of the basic procedures of PNF, the irradiation is to use manual resistance to move a strong body part without pain so that the contraction of muscles can be delivered to the body part with pain. This indirect treatment is effective in functional enhancement for the patients with musculoskeletal diseases accompanying severe pain (Voss, 1967).

In addition, PNF adopted International Classification Functioning, Disability, and Health (ICF) developed by World Health Organization (WHO) in assessing the patients for a clearer functional enhancement of the patients with activity limitations. ICF (WHO, 2001) classifies the body structure, body function, activities, and participation as the main elements of disability, while classifying personal factors and environmental factors as the supplementary elements of disability. This classification system was developed with a view to enhance the activities of patients with disability (Stucki et al., 2007).

In this study, the patients who are with limitations in activities for eating due to limitations in opening the mouth were classified for the functional disabilities of temporomandibular joints based on ICF and verify the effectiveness in enhancing the mouth opening activity of the TMD patients by restoring the abnormal alignment of the neck with using PNF programs for 4 weeks.

\section{MATERIALS AND METHODS}

\section{Participant}

The patient in this case was a 41-year-old female, working for the hospital as a physical therapy. Since 2015, the patient has complained about pain in the temporomandibular joint of left side (visual analogue scale [VAS] 6) and noise when opening and closing the mouth within the temporomandibular joint. Especially, when the patient tried to open the mouth wide to eat specific foods such as an apple, the patient felt discomfort on the right-side temporomandibular joint and subluxation. As a result, the patient developed a fear of opening the mouth wide for eating foods.

The patient also complained about pain in the neck. The profile of the patient showed a forward-headed posture, in which the head is located protruded more compared to the location of the neck, indicating an abnormal alignment of the cervical spine (Fig. 1). Neurological causes and other symptoms that cause TMD were not found in the patients, so it is ruled out the composition of the exercise program.
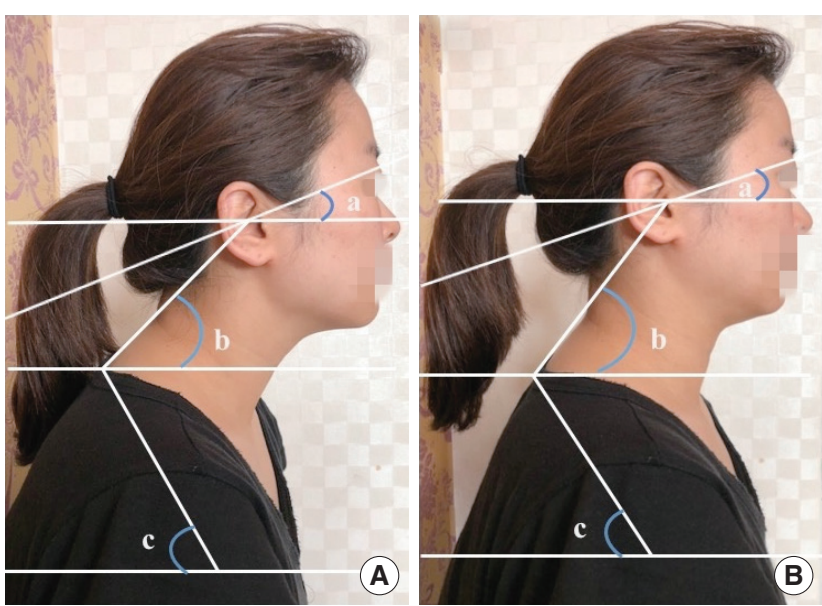

Fig. 1. Posture alignment assessment (impairments test). (A) Proprioceptive neuromuscular facilitation therapeutic exercise before the start of shooting (May 7, 2017); (B) Exercise 4 weeks elapsed after shooting (June 6, 2017); sagittal head angle (a), cervical line angle (b), and shoulder angle (c). Informed consent was obtained from the subject for the publication.

This study was conducted after receiving consent for the publication of informations and photos of patients who participated in the study.

\section{Instruments}

The instrument used in this study to measure activity limitation with opening the mouth wide and biting an apple was a 20$\mathrm{cm}$ plastic ruler (Eintec, Seoul, Korea). Before and after the doing PNF programs, the up-down length of the bite mark on the apple and the left-right width of it were measured. The length of the apple that was bitten was measured using a tape measure (Tajima, Seoul, Korea).

The test on the abnormal alignment of the cervical spine, which was designated as the impairment of body structure and body function, was conducted using a plastic goniometer (HiRes, Minnesota, MN, USA), in order to measure the angle to the cervical spine alignments.

\section{Activity limitation test}

In order to test the discomfort when eating large foods, which was the complaint the patient reported as the activity limitation, an apple that was bigger than the fully open mouth of the patient. Then, the total height and width of the bite mark were measured using a tape measure. And, for this, the average of three repeated measurements was used as the final result. 


\section{Impairment of body structure and body function test}

In order to assess the contraction of the upper cervical extensors as well as the proper alignment of the neck, the posture alignment assessment, developed by Ruivo et al. (2014) was used. With the patient standing, a photo of the sagittal plane was taken. And, with the image of the profile of the patient, the three angles that were related to the alignment of the cervical spine were measured.

These three angles were, respectively;

Sagittal head angle - The angle between the horizontal line that passes the tragus and the lateral line that connects the tragus.

Cervical line angle - The cervical line angle is used as a highly reliable indicator of forward head position. This is the angle between the horizontal line that cuts through the spinous processes of $\mathrm{C} 7$ and the line that connects the tragus.

Shoulder angle - The angle between the line that connects the center of humerus and the spinous process of $\mathrm{C} 7$ and the horizontal line that passes through the center of humerus.

\section{Procedure}

The procedure of this study was composed of four steps. The first step was the process of patient evaluation based on ICF, where the hypothesis of impairment for the activity limitation was set. The second step was the pretest of the impairment and activity limitation, while the third step was the application of the PNF exercise program to the patient. The fourth step was the posttest on the activity limitation and the impairment.

The application of the PNF program was composed of 12 sessions over 4 weeks and each session continued $30 \mathrm{~min}$. The duration and purpose of this study were agreed upon with the participating patients prior to the commencement of the study.

\section{The plan for patient assessment and the application of the PNF program}

As for the PNF, the ICF was included in the stage where the treatment plan was established, so that a hypothesis for the PNF programs for the impairment of the body structure and body function, which was the cause of the activity limitation complained about by the patient. Also in this study, the patient was interviewed before the commencement of the treatment to examine the degree of activity limitation experienced by the patient in her daily life. Especially, the patient complained about the activity limitation that the right-side chin was dislocated when she tried to bite off a large piece of food, such as an apple. Because of this, the patient complained about participation restriction in eating outside restaurant with her family.

\section{Pretest and posttest}

The pretest was conducted on the day before the commencement of the PNF program, while the posttest was conducted on the day after 4 weeks since the termination of the PNF program. All tests were conducted 3 times before commencing the treatment and 4 weeks after the treatment, and the average of the measurements was used as the result value.

\section{The PNF programs}

The PNF program was composed of a total of eight therapeutic exercises, including the home exercise. In consideration of the alignment of the cervical spine and the pain in the temporomandibular joint, the posture with a wider base of support was modified to have a narrower one. All exercise programs were designed based on the PNF basic principles, basic procedures, and philosophy of PNF.

And, the patterns and techniques of PNF were adapted to the hypothesis of activity limitation. The PNF program, which spanned

Table 1. Proprioceptive neuromuscular facilitation (PNF) programs

\begin{tabular}{|c|c|c|c|c|}
\hline Program number & Goal of exercise & Using PNF pattern and PNF tools & Patients position & Using PNF technique \\
\hline 1 & Elongation of upper cervical extensor & Flexion of short neck & Hook-lying & Contract relax \\
\hline 2 & $\begin{array}{l}\text { Reciprocal inhibition of suboccipital muscles with } \\
\text { strengthening infra-hyoid muscles }\end{array}$ & Flexion of short neck & Hook-lying & Combination of isotonics \\
\hline 3 & $\begin{array}{l}\text { Increased stability in corrected alignment of neck and } \\
\text { TMJ with spatula }\end{array}$ & Approximation & Sitting & Stabilizing reversals \\
\hline 4 & Tongue exercise in corrected posture of neck & Approximation & Sitting & Combination of isotonics \\
\hline 5 & $\begin{array}{l}\text { Increased mobility of mandible in corrected posture } \\
\text { of neck }\end{array}$ & Approximation to head and traction to mandible & Sitting & \\
\hline 6 & Opening mouth & Approximation to head & Sitting & Combination of isotonics \\
\hline 7 & Eating apple & Approximation to head and optimal resistance & Sitting & Rhythmic stabilization \\
\hline 8 & Holding the towel behind neck and opening mouth & Home exercise & Sitting & \\
\hline
\end{tabular}


Table 2. Proprioceptive neuromuscular facilitation programs of each week

\begin{tabular}{llc} 
Week & Program number & Durations (min) \\
\hline 1 & $1,2,3,4$ & 30 \\
2 & $1,2,3,4,5,8$ & 30 \\
3 & $1,2,3,4,5,6,7,8$ & 30 \\
\hline
\end{tabular}

Table 3. Apple bite measuring length (activity limitation test)

\begin{tabular}{lcc}
\hline Variable & Pre & Post \\
\hline Length of up and down $(\mathrm{cm})$ & $4.23 \pm 0.06$ & $5.25 \pm 0.07$ \\
Length of left and right $(\mathrm{cm})$ & $2.40 \pm 0.16$ & $3.43 \pm 0.02$ \\
\hline
\end{tabular}

Values are presented as mean \pm standard deviation.

over 4 weeks, had the programs of each week adjusted by combining three to four exercise programs in accordance with the level of adaptation to the regime by the patient. As a result, three sessions were conducted in each week, and each of these sessions lasted for 30 min (Tables 1, 2). Before the starting of the exercise program, the patient was given with an overall explanation and detailed instructions on deep-breathing. And, the exercise was interrupted when the patient reported pain or fatigue to have a 2- to 3-min break. The break was not included in the total duration of exercises.

\section{Data analysis}

The data collected from the test were visually presented for analysis. The data before and after the PNF were plotted using MS Office Excel 2013. All measurements were taken 3 times and averaged to be taken as the result

\section{RESULTS}

\section{Results of the apple bit measuring length: Activity limitation test}

For the test, the patient was instructed to take a stable posture. Then, an apple was given to her, which her bit off. Then, the bite mark's height and width were measured. The measurement was $4.23 \pm 0.06 \mathrm{~cm}$ before the PNF exercise program and $5.25 \pm 0.07$ $\mathrm{cm}$ after the exercise. The width measured $2.40 \pm 0.16 \mathrm{~cm}$ at the pre-test and $3.43 \pm 0.02 \mathrm{~cm}$ at the post test. The condition of the patient improved as shown here, as it was confirmed that the patient was now capable of opening her mouth wider to bite off the apple (Table 3).

\section{Results of the posture alignment assessment and VAS: impairment test}

The therapist used posture alignment assessment in order to as-
Table 4. Posture alignment assessment (impairments test)

\begin{tabular}{lcc}
\hline Variable & Pre & Post \\
\hline Sagittal head angle $\left(^{\circ}\right)$ & $18.58 \pm 0.38$ & $21.25 \pm 0.26$ \\
Cervical line angle $\left(^{\circ}\right)$ & $45.90 \pm 0.36$ & $50.41 \pm 0.68$ \\
Shoulder angle & $59.80 \pm 0.43$ & $53.93 \pm 0.31$ \\
VAS (point) & 6 & 2
\end{tabular}

Values are presented as mean \pm standard deviation.

VAS, visual analogue scale.

sess the alignment of the neck and the contraction of the upper cervical extensors. The results showed that the sagittal head angle was $18.58^{\circ} \pm 0.38^{\circ}$ at the pretest and $21.25^{\circ} \pm 0.26^{\circ}$ after the PNF program, indicating that the position of the head as per the alignment of the neck changed. The cervical line angle measured $45.90^{\circ} \pm 0.36^{\circ}$ at the pretest and $50.41^{\circ} \pm 0.68^{\circ}$ after PNF, indicating improvement in the alignment of the neck and extension of the upper cervical extensors. The shoulder angle measured $59.80^{\circ} \pm 0.43^{\circ}$ during the pretest and $53.93^{\circ} \pm 0.31^{\circ}$ after PNF, indicating an improvement with the alignment of the shoulder (Fig. 1, Table 4). The level of pain experienced by the patient when she opened the mouth measured using VAS scored 6 out of 10 initially but later decreased to 2 after the PNF program (Table 4).

\section{DISCUSSION}

The causes of TMD reported in the literature varied widely, including improper chewing or other bad habits, trauma, improper postures, spinal deformation, or mental stress (Clark et al., 1989). Okeson (2015) also reported that TMD may be cause not only by the structural defects around the temporomandibular joint but also abnormal alignment of the neck or deformation, resulting in anomaly in the temporomandibular joint. This argument supports the approach of Janda, who suggested that the structural damages in the surrounding tissues caused during the inflammatory phase after an injury is inflicted on the musculoskeletal system (Key et al., 2008; Morris et al., 2006). The forward headed posture, which was the hypothesis of the TMD of the patient, causes excessive tension in certain muscles in the head and neck, undermining vertical stability around the head and neck. The forward headed posture could be extending the hyoid muscles, while pulling the mandible to the lower, backward direction. the mandibular fossa and mandibular condyle are dislocated, so that the articular disc at the temporomandibular joint was obstructed, causing impairing the chewing and eating activities (Paesani et al., 1999). Such an abnormal movement causes inflammation in the temporomandibular joint, making noises and causing pain 
when opening the mouth.

PNF, which is based on the philosophy that all human beings have the undeveloped potential that could be used for treatment can be used comprehensively at the physical and mental level on patients with musculoskeletal impairments, such as TMD. The philosophy of PNF is to address not only the physical issues of the patients with impairments in body structures and body functions causing pain or activity limitation but also use the total body approach and the positive approach to focus on improving the activity level and the body structures and body functions (Angeli et al., 2018; Hruby, 1985). In this case, too, the philosophy of PNF and ICF were used as the basis in addressing the activity limitation of the patient, who could not open her mouth wide enough to eat larger foods.

The medical history of the patient, observation, and tests were used in order to develop a hypothesis on the activity limitation of the cervical spine due to shortening of upper cervical extensors which was again caused by the forward headed posture. Based on these, the activity limitation test and the impairment of body structure and body function test were conducted before and after the PNF program. The posture alignment assessment used in the patient's impairment test showed that the angle of the cervical line angle was $43^{\circ}$, which means that the patient had a forward headed posture. In addition, the patient had an activity limitation that could not open the mouth to bite apple.

The patient had a deep breathing exercise at the supine position before commencing the PNF therapeutic exercise. This breathing exercise has been reported to be effective in relaxing the muscle that tensed up due to pain (Busch et al., 2012). The first PNF program was to apply the hold-relax method on the infra-hyoid muscles in order for elongation and relaxation of the upper cervical extensors with using the flexion of neck patterns. The chin-in movement was applied using the combination of isotonics to reinforce elongation of the upper cervical extensors and infra hyoid muscles. De Wijer et al. (1996) suggested that it is necessary to assess and treat the cervical spine and shoulder girdle in addition to the stomatognathic system when assessing the TMD patients, in order to help alleviating the symptoms of the head and neck, which are complex and persistent. Kim and Park (2016), in their study of applying stabilizing reversals and rhythmic stabilization for trunk stability, reported that rhythmic stabilization facilitated balance and stability. For the TMD patient in this case, also, the stabilization technique of PNF was applied in order to achieve stability around the temporomandibular joint before the mouth-opening exercise. The fourth PNF program was given with the patient in a sitting position, with the neck and spine aligned straight. The therapist applied approximation on the top of the head while using the other hand to hold the tongue of the patient using gauze gently and gave resistance to the outside of the mouth, in order to induce a movement of pulling the tongue back into the mouth. The PNF technique used here is combination of isotonics, which reinforces contraction and strengthening of the agonistic muscle. Yagi et al. (2008) focused on the tongue movement of the TMD patients and reported that frequent hanging out the tongue may cause impairment of the temporomandibular joint. And, with the pulling of the tongue, they tried to realign the cervical spine and relax the muscles surrounding the temporomandibular joint (de Félicio et al., 2007). The seventh PNF program was the activity level exercise, where the neck and jaw of the patient were aligned and the therapist contacted the patient's head and shoulder of the patient with bare hand, applying resistances and approximations in various directions, in applying the rhythmic stabilization technique for the neck and trunk stability.

The eighth PNF program was composed of the home exercises that the patient could perform by herself at home. This was to maintain the corrective effect on the forward headed posture, which was hypothesized as the contraction of the upper cervical extensors for the activity limitation. De Freitas et al. (2013) reported that the home programs for TMD were effective in addressing the problems of the patients who were closely related to improper habits.

In this study, the impairment of the patient, that is, having activity limitation in opening the mouth wide to eat bigger foods, was determined, and the manual contact at the temporomandibular joint, where the pain originated, was replaced by a more indirect therapy of moving the trunk of the patient to align the cervical spine and mitigating the pain through a breathing exercise. The alignment of cervical spine is one of the key elements of remedying the location of the disc at the temporomandibular joint. After a 4-week PNF therapeutic exercises, the patient showed an improvement that she could now open her mouth wide to eat bigger food without pain. The 4-week PNF programs, the proper PNF techniques were chosen in order to enhance the stability and mobility of the temporomandibular joint based on the existence of pain. With this, the impairment in the body structure and body function was improved.

Therefore in the follow-up study, more TMD patients would need to be included in order to verify the effect of the therapeutic exercise programs suggested herein. And, the subsequent study 
topic should be the development of the home program in order to maintain the effect of the therapeutic exercise for the TMD patient and quantification of the strength of the manual resistance of the therapist for a study using PNF therapeutic exercises.

\section{CONFLICT OF INTEREST}

No potential conflict of interest relevant to this article was reported.

\section{REFERENCES}

Angeli JM, Harpster KL, Hanson E, Sheehan A, Schwab SM. Patient- and caregiver-identified preferences: Dimensions of change in developmental therapy treatment goals. Dev Neurorehabil 2018:1-8 [Epub]. https://doi.org/10.1080/17518423.2018.1425754.

Busch V, Magerl W, Kern U, Haas J, Hajak G, Eichhammer P. The effect of deep and slow breathing on pain perception, autonomic activity, and mood processing--an experimental study. Pain Med 2012;13:215228.

Clark GT, Seligman DA, Solberg WK, Pullinger AG. Guidelines for the examination and diagnosis of temporomandibular disorders. J Craniomandib Disord 1989;3:7-14.

Cortese S, Mondello A, Galarza R, Biondi A. Postural alterations as a risk factor for temporomandibular disorders. Acta Odontol Latinoam 2017; 30:57-61.

de Félicio CM, Freitas RL, Bataglion C. The effects of orofacial myofunctional therapy combined with an occlusal splint on signs and symptoms in a man with TMD-hypermobility: case study. Int J Orofacial Myology 2007;33:21-29.

de Freitas RF, Ferreira MÂ, Barbosa GA, Calderon PS. Counselling and self-management therapies for temporomandibular disorders: a systematic review. J Oral Rehabil 2013;40:864-874.

de Wijer A, Steenks MH, de Leeuw JR, Bosman F, Helders PJ. Symptoms of the cervical spine in temporomandibular and cervical spine disorders. J Oral Rehabil 1996;23:742-750.
El Hage Y, Politti F, Herpich CM, de Souza DF, de Paula Gomes CA, Amorim CF, de Oliveira Gonzalez T, Biasotto-Gonzalez DA. Effect of facial massage on static balance in individuals with temporomandibular disorder - a pilot study. Int J Ther Massage Bodyw 2013;6:6-11.

Hruby RJ. The total body approach to the osteopathic management of temporomandibular joint dysfunction. J Am Osteopath Assoc 1985; 85:502-510.

Kabat H, Knott M. Proprioceptive facilitation technics for treatment of paralysis. Phys Ther Rev 1953;33:53-64.

Key J, Clift A, Condie F, Harley C. A model of movement dysfunction provides a classification system guiding diagnosis and therapeutic care in spinal pain and related musculoskeletal syndromes: a paradigm shift-Part 1. J Bodyw Mov Ther 2008;12:7-21.

Kim JJ, Park SY. Immediate effects of the trunk stabilizing exercise on static balance parameters in double-leg and one-leg stances. J Phys Ther Sci 2016;28:1673-1675.

Morris CE, Greenman PE, Bullock MI, Basmajian JV, Kobesova A. Vladimir Janda, MD, DSc: tribute to a master of rehabilitation. Spine (Phila Pa 1976) 2006;31:1060-1064.

Okeson JP. Evolution of occlusion and temporomandibular disorder in orthodontics: Past, present, and future. Am J Orthod Dentofacial Orthop 2015;147(5 Suppl):S216-223.

Paesani D, Salas E, Martinez A, Isberg A. Prevalence of temporomandibular joint disk displacement in infants and young children. Oral Surg Oral Med Oral Pathol Oral Radiol Endod 1999;87:15-19.

Ruivo RM, Pezarat-Correia P, Carita AI. Cervical and shoulder postural assessment of adolescents between 15 and 17 years old and association with upper quadrant pain. Braz J Phys Ther 2014;18:364-371.

Stucki G, Cieza A, Melvin J. The International Classification of Functioning, Disability and Health (ICF): a unifying model for the conceptual description of the rehabilitation strategy. J Rehabil Med 2007;39:279285.

Voss DE. Proprioceptive neuromuscular facilitation. Am J Phys Med 1967; 46:838-899.

Yagi S, Fukuyama E, Soma K. Involvement of sensory input from anterior teeth in deglutitive tongue function. Dysphagia 2008;23:221-229. 